A\&A 389, 513-518 (2002)

DOI: $10.1051 / 0004-6361: 20020589$

(c) ESO 2002

\title{
The Eagle's EGGs: Fertile or sterile?
}

\author{
M. J. McCaughrean and M. Andersen
}

\author{
Astrophysikalisches Institut Potsdam, An der Sternwarte 16, 14482 Potsdam, Germany \\ e-mail: mjm@aip.de;mortena@aip.de
}

Received 23 January 2002 / Accepted 16 April 2002

\begin{abstract}
We present a deep, high spatial resolution $(0.35 \operatorname{arcsec} F W H M)$, near-infrared $(1-2.5 \mu \mathrm{m})$ imaging survey of the Eagle Nebula, M 16, made with the VLT, centred on the famous elephant trunks. We compare these data with the existing HST optical images to search for evidence of ongoing or recent star formation in the trunks, and in particular in the 73 small evaporating gaseous globules (EGGs) on their surface. We find that two of the three HST trunks have relatively massive YSOs in their tips. Most of the EGGs appear to be empty, but some $15 \%$ of them do show evidence for associated young low-mass stars or brown dwarfs: in particular, there is a small cluster of such sources seen at the head of the largest trunk.
\end{abstract}

Key words. stars: formation - stars: pre-main sequence - stars: low-mass, brown dwarfs - H II regions infrared: stars

\section{Introduction}

The life history of a star appears to be determined both by "nature", imprints received prior to birth, and "nurture", influences from the environment surrounding it in the early years after birth. Crucial amongst these external influences is the presence of massive stars: their intense ionising flux and strong winds can turn the common parental molecular cloud into an H II region and thus terminate star formation, as well as evaporate circumstellar disks around low-mass stars and prevent planet formation (Hollenbach et al. 2000). On the other hand, massive stars can also lead to radiative implosion of surrounding molecular cores, initiating secondary star formation (Larosa 1983; Bertoldi 1989; Lefloch \& Lazareff 1994).

A famous example of the effects wrought by massive stars is seen in the Eagle Nebula, M 16. OB stars in the young stellar cluster NGC 6611 (Hillenbrand et al. 1993) are photoionising surrounding molecular material, leading in one region to the creation of three elongated columns or "elephant trunks", as detailed in the iconic Hubble Space Telescope images of Hester et al. (1996; hereafter H96). H96 identified 73 small (typically $\sim 0.5$ arcsec or $1000 \mathrm{AU}$ diameter) protrusions on or near the surface of the trunks, which they dubbed EGGs, for evaporating gaseous globules. Noting a few apparent associations with stars seen in the HST and/or near-infrared images (Hillenbrand et al. 1993), H96 proposed that the EGGs are created as the

Send offprint requests to: M. J. McCaughrean, e-mail: mjm@aip.de
NGC 6611 ionisation front sweeps over dense condensations of molecular material harbouring young stars, i.e., they might also be considered as "eggs". These embedded stars would be exposed prematurely as the surrounding EGG is evaporated, thus removing the reservoir for future accretion and perhaps also the circumstellar disk. H96 suggested that if most stars form under the influence of massive stars, this influence might then ultimately determine important results such as the stellar initial mass function and the fraction of stars with planetary systems.

However, missing in this hypothesis is direct observational evidence that the M 16 columns and EGGs indeed contain a substantial population of young stars. As the optical images of H96 were not able to probe the dusty interiors of the columns, longer wavelength data are required. The region has been surveyed in the near-infrared by Walsh \& White (1982), Chini et al. (1992), Hillenbrand et al. (1993), Currie et al. (1996), and McCaughrean (1997), in the mid-infrared by Pilbratt et al. (1998), and in the millimetre by Pound (1998) and White et al. (1999). However, none of these studies had the required combination of high spatial resolution, sensitivity, and field-ofview to provide a detailed view of possible ongoing star formation in the trunks, or to carry out a statistically meaningful survey for embedded sources in the EGGs.

Two recent near-infrared imaging studies (Sugitani et al. 2002, hereafter S02; Thompson et al. 2002, hereafter TSH02) have revisited the region with better spatial resolution and sensitivity, and both describe evidence for massive star formation in the tips of two of the trunks, as also seen in the previous images of Hillenbrand et al. (1993) and McCaughrean (1997). However, neither study 
directly addressed the question of star formation in the EGGs. We have used the VLT to make a new near-infrared survey of a large region containing NGC 6611, the elephant trunks, and their EGGs, with sufficient spatial resolution $(\sim 0.35$ arcsec $F W H M)$ and sensitivity $\left(K_{\mathrm{s}}>20^{\mathrm{m}}\right)$ to probe EGGs individually. Here we present just the data covering the elephant trunks and our essential findings concerning the EGGs: the entire data set can be seen in McCaughrean \& Andersen (2001), and a detailed analysis will be given in a subsequent paper.

\section{Observations}

The data were obtained with the facility near-infrared camera/spectrograph, ISAAC, on the 8.2-m VLT UT1 Antu in service mode (ESO program 67.C-0595) on April 10 and May 8-10 2001. The ISAAC short-wavelength camera has a $1024 \times 1024$ pixel $\mathrm{HgCdTe} H A W A I I$ array covering $2.5 \times 2.5$ arcmin at $0.147 \mathrm{arcsec} /$ pixel. A slightlyoverlapping $4 \times 4$ position mosaic was made to cover a $\sim 9 \times 9$ arcmin region centred on the elephant trunks and including much of NGC 6611: the inner $2 \times 2$ mosaic was imaged repeatedly to go deep on the most critical region, while the outer part of the mosaic was covered less often, to provide sky data and a broader survey. Data were taken in three broad-band filters $J_{\mathrm{s}}(1.24 \mu \mathrm{m}), H(1.65 \mu \mathrm{m})$, and $K_{\mathrm{s}}(2.16 \mu \mathrm{m})$. A standard observing block involved imaging each position in the central $2 \times 2$ mosaic three times and for $100 \mathrm{~s}$ each time (10 coadds of $10 \mathrm{~s})$; the outer ring of 12 positions in the $4 \times 4$ position mosaic was imaged once for $50 \mathrm{~s}$ ( 5 coadds of $10 \mathrm{~s})$.

Data reduction was standard. For any given image, a blank sky frame was constructed by making a median stack of the other 23 images within the same block. A clean sky frame was always achieved, despite the extremely crowded nature of the region. This sky frame was subtracted from the image and the result then flat-fielded using differential twilight flats (i.e., bright sky minus faint sky). Corrections were made for the small geometric distortion in ISAAC before the images were aligned, intensity normalised, and co-added to form three final mosaics, one per filter.

In $H$ and $K_{\mathrm{s}}$, data from only the better of two observing blocks were used, while in $J_{\mathrm{s}}$, data from four equalquality blocks were used. Thus for the deepest, central section of the mosaics discussed here, the effective integration time per pixel is $300 \mathrm{~s}$ in $H$ and $K_{\mathrm{s}}$, and $1200 \mathrm{~s}$ in $J_{\mathrm{s}}$, the longer integration time at $J_{\mathrm{s}}$ in part compensating for higher extinction in the EGGs at the shorter wavelength. Spatial resolution in the coadded mosaics is $0.38,0.36$, and $0.33 \operatorname{arcsec} F W H M$ in $J_{\mathrm{s}}, H$, and $K_{\mathrm{s}}$, respectively. Photometric calibration via faint standard stars yields detection limits (defined as a star whose brightest pixel is detected at $3 \sigma$ above the local noise) at $J_{\mathrm{s}}, H$, and $K_{\mathrm{s}}$ of $22^{\mathrm{m}} \cdot 6,21^{\mathrm{m}} \cdot 3$, and $20^{\mathrm{m}} \cdot 4$, respectively. In regions of bright nebular emission, these limits are somewhat reduced.

Figure 1 shows the region centred on the trunks in a three-colour composite. This region and the complete survey mosaic can be seen at full resolution in McCaughrean \& Andersen (2001).

\section{Results}

The contrast between the HST optical emission-line image of H96 and the VLT near-infrared broad-band image is striking. The HST image is dominated by the three gas and dust columns: the VLT image shows not only these, but also a huge number of stars towards, in, and beyond M 16. Stars seen in the VLT image as blue are in M 16 or in the foreground, but the great majority of the stars are fainter and yellow, and are background field stars seen along the line-of-sight through the bulge towards the inner galaxy $\left(l, b \sim 17^{\circ},+1^{\circ}\right)$, through the molecular material out of which M 16 was created. An infrared colour-colour diagram derived from our full data set and containing some $5 \times 10^{4}$ stars shows this background screen to have an extinction of $A_{V} \sim 10^{\mathrm{m}}$. There are also a number of orange and red sources associated with the columns and thus seen through additional dust extinction. However, simple visual examination cannot distinguish between stars embedded in the trunks or background stars seen through them.

The three columns are also considerably changed in the near-infrared, as noted and discussed by S02 and TSH02. The heads of the three columns are slightly reduced in size with respect to the optical images, but all retain significant opaque cores. The largest column (C1) disappears almost completely along much of its length below the head (cf. Pound 1998), and the middle trunk (C2) is also broken in the middle: only the smallest trunk (C3) remains continuous.

\subsection{Star formation in the columns}

The immediate goal of our VLT experiment was to enable a sensitive search for embedded young stars in the EGGs. To characterise any such objects seen in our images, we use the pre-main sequence models of Baraffe et al. (1998) at $\leq 1 M_{\odot}$ and Palla \& Stahler (1999) at higher masses. To generate magnitudes for the ISAAC $J_{\mathrm{s}}$ and $K_{\mathrm{s}}$ filters, we have interpolated those available for the standard $J$, $H$, and $K$ filters according to band centre, a justifiable approximation given the close similarity of the two filter sets. We assume a distance of $1.9 \mathrm{kpc}$ to M 16 and $R=3.75$ for its extinction law (Hillenbrand et al. 1993), and a generic age of 1 Myr for all putative young embedded sources. Under these assumptions, our VLT detection limits correspond to a limiting mass of $<0.01 M_{\odot}\left(10 M_{\mathrm{Jup}}\right)$ in all three filters, assuming no extinction. However, since we are searching for sources embedded in the EGGs, we calculate that a $0.075 M_{\odot}$ source at the star:brown dwarf boundary can be detected through up to $A_{V} \sim 16^{\mathrm{m}}$, $23^{\mathrm{m}}, 30^{\mathrm{m}}$ of extinction at $J_{\mathrm{s}}, H$, and $K_{\mathrm{s}}$, respectively, for an $R=3.75$ extinction law (Cardelli et al. 1989). Assuming an embedded star lies in the centre of its EGG, then the 0.5 arcsec median diameter of an EGG converts 


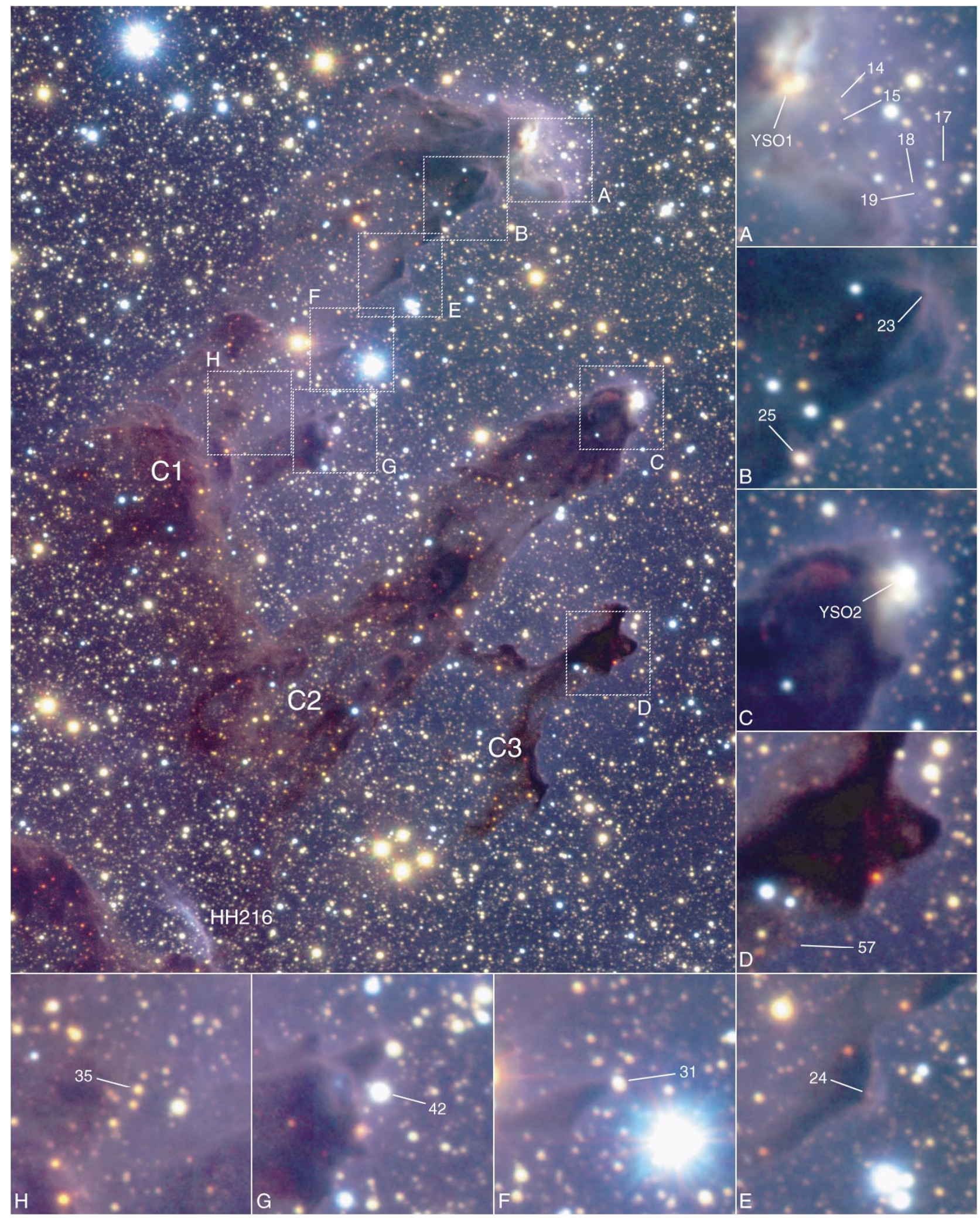

Fig. 1. True-colour near-infrared $(1-2.5 \mu \mathrm{m})$ image of the M 16 elephant trunks. The $J_{\mathrm{s}}$ data are shown as blue, $H$ as green, and $K_{\mathrm{s}}$ as red. The cube root of the intensities were taken to compress the dynamic range before normalising and combining the three mosaics. The main image is centred at $18^{\mathrm{h}} 18^{\mathrm{m}} 52.7^{\mathrm{s}},-13^{\circ} 50^{\prime} 09^{\prime \prime}(\mathrm{J} 2000.0)$ and covers $158 \times 214$ arcsec $(1.5 \times 2.0 \mathrm{pc}$ at $1.9 \mathrm{kpc}$ ); north is up, east left. The subimages have been magnified by a factor of 2.9 and each covers $18.5 \times 18.5$ arcsec $(0.17 \times 0.17 \mathrm{pc})$. Labels mark EGGs from H96 found to be associated with point sources as described in the text; E23, an EGG with no near-infrared point source, but thought to contain an embedded protostar driving a collimated jet; YSO1 and YSO2, massive sources in the tips of $\mathrm{C} 1$ and $\mathrm{C} 2$, respectively; and $\mathrm{HH} 216$, an optically-visible Herbig-Haro object. Due to the large dynamic range, some of the very faintest sources are not easily seen in these subimages, but can be seen in the original data. 
to a column of $7 \times 10^{15} \mathrm{~cm}$ to the source. To yield an extinction of $A_{V}=30^{\mathrm{m}}$, the mean volume density would then have to be $\sim 10^{7} \mathrm{~cm}^{-3}, \sim 50$ times greater than is measured for the heads of the trunks (White et al. 1999; Pound 1998). Therefore, despite a likely above average density in the EGGs, the present data appear to have the sensitivity required to probe most of the EGGs to the brown dwarf limit and below.

Without classification spectroscopy, only crude mass estimates can be made for detected sources, by placing them in the infrared colour-magnitude diagram and dereddening until they intercept the model 1 Myr isochrone. As some sources will show excess long-wavelength emission in the colour-colour diagram indicative of a circumstellar disk, the $J_{\mathrm{s}}$ versus $\left(J_{\mathrm{s}}-H\right)$ colour-magnitude diagram is generally used for the mass estimation. It is clear that this method is approximate at best, and the results presented in Table 1 should be taken only as an indication of the likely masses of the embedded sources, in lieu of further observations.

\subsubsection{Massive YSOs in the columns}

The tips of both $\mathrm{C} 1$ and $\mathrm{C} 2$ show evidence for bright embedded young stellar objects (YSOs). Both were detected in earlier infrared images (Hillenbrand et al. 1993; McCaughrean 1997), and have been described in detail by $\mathrm{S} 02$ and TSH02. The source in $\mathrm{C} 1$ (here YSO1; P1 of S02; M16ES-1 of TSH02) is very red and sits at the southern tip of a complex extended nebula bisected by a lane of extinction associated with one of the more prominent "towers" on the head of $\mathrm{C} 1$. It is also detected at 7-11 $\mu \mathrm{m}$ by MSX (Price et al. 2001), $12-18 \mu \mathrm{m}$ by ISO (Pilbratt et al. 1998), and as a bright hard X-ray source by Chandra (Gagné, personal communication).

Assuming the $J_{\mathrm{s}}$ and $H$ fluxes (see Table 1 ) of YSO1 to be photospheric, we deduce an extinction of $A_{V} \sim$ $27^{\mathrm{m}}$ and a dereddened absolute $M_{H}$ of $-1^{\mathrm{m}} \cdot 6$, consistent with a $10 M_{\odot}$ ZAMS star with a spectral type of B1 (Schmidt-Kaler 1982). However, integrating under near-, mid-, and far-infrared fluxes, TSH02 derived a luminosity of $\sim 200 L_{\odot}$, equivalent to only a $4 M_{\odot}$ ZAMS star and closer to late B in spectral type. TSH02 also found no evidence for the coincident $\mathrm{Pa} \alpha$ emission from ionised gas that would be expected from even a late B star, and thus, as they note, it is possible that YSO1 is perhaps a compact cluster or, in our opinion more likely, a young protostar. The $2.7 \mathrm{~mm}$ continuum emission reported by $\mathrm{S} 02$ might, for example, be free-free emission from an associated ultracompact H II region rather than a disk, perhaps supporting the young, high-mass protostar hypothesis.

The tip of $\mathrm{C} 2$ also contains a bright point source (here YSO2; T1 of S02, M16ES-2 of TSH02) illuminating a compact reflection nebulosity. S02 claim that this nebulosity is bipolar, but instead we see a fainter second source roughly 1 arcsec south, and which we find to be consistent with a lightly reddened field star (see also Fig. 10b of H96).
Table 1. Photometry and derived properties for sources embedded in the trunks and EGGs assuming 1 Myr ages for all sources.

\begin{tabular}{lcccccc}
\hline \hline Source $^{\mathrm{a}}$ & $J_{\mathrm{s}}{ }^{\mathrm{b}}$ & $H^{\mathrm{b}}$ & $K_{\mathrm{s}}^{\mathrm{b}}$ & $\begin{array}{c}\text { Mass }^{\mathrm{c}} \\
{\left[M_{\odot}\right]}\end{array}$ & $\begin{array}{c}A_{V}{ }^{\mathrm{c}} \\
{[\mathrm{mag}]}\end{array}$ & Excess $^{\mathrm{d}}$ \\
\hline YSO1 & $17^{\mathrm{m}} \cdot 8$ & $14^{\mathrm{m}} \cdot 7$ & $12^{\mathrm{m}} \cdot 9$ & 10 & 27 & Yes \\
YSO2 & $15^{\mathrm{m}} \cdot 2$ & $13^{\mathrm{m}} \cdot 8$ & $12^{\mathrm{m}} \cdot 8$ & $2-6$ & 15 & Yes \\
E14 & $20^{\mathrm{m}} \cdot 0$ & $18^{\mathrm{m}} \cdot 8$ & $17^{\mathrm{m}} \cdot 7$ & 0.04 & 7 & Yes \\
E15 & $22^{\mathrm{m}} \cdot 5$ & $20^{\mathrm{m}} \cdot 7$ & $19^{\mathrm{m}} \cdot 8$ & 0.03 & 12 & No \\
E17 & $20^{\mathrm{m}} \cdot 7$ & $19^{\mathrm{m}} \cdot 9$ & $18^{\mathrm{m}} \cdot 6$ & 0.02 & 3 & Yes \\
E18 & $19^{\mathrm{m}} \cdot 3$ & $19^{\mathrm{m}} \cdot 5$ & $18^{\mathrm{m}} \cdot 8$ & $(0.04)$ & $(9)$ & Yes \\
E19 & $19^{\mathrm{m}} \cdot 1$ & $19^{\mathrm{m}} \cdot 0$ & $18^{\mathrm{m}} \cdot 2$ & $(0.05)$ & $(5)$ & Yes \\
E24 & $20^{\mathrm{m}} \cdot 7$ & $19^{\mathrm{m}} \cdot 7$ & $18^{\mathrm{m}} \cdot 4$ & 0.02 & 5 & Yes \\
E25 & $16^{\mathrm{m}} \cdot 9$ & $15^{\mathrm{m}} \cdot 3$ & $13^{\mathrm{m}} \cdot 8$ & 0.5 & 9 & Yes \\
E31 & $17^{\mathrm{m}} \cdot 6$ & $15^{\mathrm{m}} \cdot 9$ & $14^{\mathrm{m}} \cdot 6$ & 0.35 & 10 & Yes \\
E35 & $20^{\mathrm{m}} \cdot 0$ & $17^{\mathrm{m}} \cdot 1$ & $15^{\mathrm{m}} \cdot 6$ & 0.95 & 22 & No \\
E42 & $14^{\mathrm{m}} \cdot 4$ & $13^{\mathrm{m}} \cdot 4$ & $12^{\mathrm{m}} \cdot 9$ & 1.0 & 4 & No \\
E57 & $>23^{\mathrm{m}} \cdot 0$ & $21^{\mathrm{m}} \cdot 1$ & $19^{\mathrm{m}} \cdot 3$ & $(0.07)$ & $(25)$ & No \\
\hline
\end{tabular}

${ }^{a}$ YSO1 and YSO2 are the sources embedded in the tips of $\mathrm{C} 1$ and $\mathrm{C} 2$ as described in the text; Enn are EGGs (see Fig. 8 of H96).

${ }^{\mathrm{b}}$ Photometry in $0.45^{\prime \prime}$ diameter aperture, with aperture correction.

${ }^{\mathrm{c}}$ Mass and extinction were calculated from $J_{\mathrm{s}}$ and $H$, apart from those in parentheses which used $H$ and $K_{\mathrm{s}}$.

${ }^{\mathrm{d}}$ Excess long-wavelength emission in the $\left(J_{\mathrm{s}}-H\right)$ vs. $\left(H-K_{\mathrm{s}}\right)$ diagram.

Again assuming photospheric emission for YSO2, we deduce an extinction of $\sim 15^{\mathrm{m}}$ and a mass of $\sim 2-5 M_{\odot}$. The non-monotonic mass-luminosity relation for young, higher-mass sources yields this ambiguity for YSO2, but we note that the luminosity of $\sim 20 L_{\odot}$ derived by TSH02 corresponds to a 2-3 $M_{\odot}$ star at 1 Myr (Palla \& Stahler 1999).

By contrast, C3 has no embedded sources in its tip, although a pair of relatively bright sources sit in the "bowl" just above it. TSH02 suggest that these may be young stars recently revealed as the tip of C3 was evaporated away, but from our wider-field images, it appears equally plausible they are simply field stars: the chances of such a pair being projected there is small, but not vanishingly so. Finally, to the south-east of the HST trunks and outside the image shown here (see McCaughrean \& Andersen 2001), there is another similar structure that has not yet been overrun by the ionisation front. The VLT images show an extended red reflection nebula in the tip of this column, which Andersen et al. (2002) have suggested to be the driving source of the Herbig-Haro flow HH 216, part of which is seen as the blue nebulous arc near the base of Fig. 1.

\subsubsection{Star formation in the EGGs}

The key issue here, previously addressed briefly by McCaughrean (1997), is to assess statistically whether or not the EGGs, as a population of small dense cores, 
are indeed forming young stars. To test this hypothesis, we first registered the HST optical and VLT near-infrared frames using 60 stars common to both: the fit has an RMS error of 0.05 arcsec, accurate enough when compared with the median EGG diameter of 0.5 arcsec. Using a list of coordinates and dimensions supplied by Jeff Hester, we examined each EGG in the HST $\mathrm{H} \alpha$ data, the individual VLT infrared mosaics, and the infrared colour composite. This process is subjective to an extent, as was the original definition of the EGGs: some are well defined and partly or completely detached from the surface of the trunks, but most are mere protrusions above the local surface. In these cases, we made a rough judgement of the size of the underlying EGG based on its apparent radius of curvature, and then searched within those boundaries.

In the 73 EGGs, we find 11 that show definite evidence for an associated near-infrared point source (Table 1). Two of these (E31, E42) sit right at the tips of "minitrunks" near the base of $\mathrm{C} 1$ and are optically visible, as noted by H96, but the remaining 9 (E14, E15, E17, E18, E19, E24, E25, E35, E57) are only visible at near-infrared wavelengths. Using the method and assumptions given above, we find that four of the sources (E25, E31, E35, E42) appear to have stellar masses in the range $0.3-1 M_{\odot}$, while the remainder (E14, E15, E17, E18, E19, E24, E57) are substellar, with masses in the range $0.02-0.07 M_{\odot}$. Remarkably, most of these substellar sources (E14-E19) are associated with rather small EGGs located at the tip of $\mathrm{C} 1$, near the much more massive YSO1. The apparent lack of low-mass stars in this region may simply be a selection effect: somewhat higher-mass sources evolve more rapidly and may have already escaped their EGGs. In this case, we would not have picked them out against the field stars according to the present EGG-based search criteria.

Another 5 EGGs show tentative evidence for embedded sources (E16, E20, E45, E46, E60), while a further 6 EGGs show near-infrared sources nearby, but on various grounds we consider these to be unrelated (E03, E23, E44, E50, E64). The remaining 51 EGGs show no evidence for associated sources in our data, including E01, which H96 suggested was coincident with a bright near-infrared source in the data of Hillenbrand et al. (1993): the VLT data clearly show it to be a background field star lying off the EGG. A more detailed discussion of each EGG will be given in our subsequent paper.

Given the huge density of background field stars in the region, some sources apparently coincident with EGGs may simply be chance projections. We can approach this problem in two ways. First, plain field stars should have normal reddened near-infrared colours, while young lowmass sources should show infrared excess emission typical of a circumstellar disk. The sources associated with E14, E17, E18, E19, E24, E25, and E31 do indeed show infrared excess confirming them as young, but the absence of excess emission from the other candidates should not necessarily be taken as evidence against them, as only half of all YSOs in Taurus-Auriga with excess $10 \mu \mathrm{m}$ emission also show it in their near-infrared colours (Kenyon \& Hartmann 1995).
Longer wavelength high spatial resolution imaging would provide crucial additional confirmation, although the required sensitivity may mean waiting until the NGST is available.

Second, we have taken advantage of the wide field covered by our data to examine the field population away from the trunks and count the number of stars per magnitude bin per unit area. For each star seen against an EGG, we can then calculate the chances of there being a field star projected within the confines of the EGG at that measured magnitude, after accounting for the additional extinction introduced by the EGG. We find this correction to be negligibly small in almost all cases, as will be discussed in our subsequent paper.

\section{Conclusions}

Our VLT images of the M16 elephant trunks show clear evidence for recent star formation. There are relatively massive, embedded YSOs at the tips of two of the HST columns, as also discussed by S02 and TSH02, and in one further column to the south-east. Furthermore, the VLT data have been able to demonstrate that while the majority of the Eagle's EGGs are sterile, at least some are fertile. That is, some $15 \%$ of them appear to be real "eggs", hatching new stars or brown dwarfs. This fraction is no larger than that found by McCaughrean (1997), despite the significantly improved data. The main reason is that several previously-claimed coincidences disappeared as the spatial resolution improved, only to be replaced by new discoveries at higher sensitivity.

This $15 \%$ must still be considered a lower limit however, as some of the EGGs remain opaque even at nearinfrared wavelengths. A case in point is E23, seen against the darkest part of $\mathrm{C} 1$ (Fig. 1). There is no associated near-infrared point source, but both infrared and optical images show a linear jet-like feature extending to the north, ending in a nebular knot, with a counter "bowshock" an equal distance to the south. This circumstantially implies the presence of a very deeply embedded protostar within E23, driving an outflow. Also, there may be other young stars in and around the columns unrelated to EGGs. Infrared excess emission can be used to identify sources of this kind: S02 found several additional candidate YSOs in this manner, and we shall return to this question in our subsequent paper.

Finally, for all our focus on the trunks and EGGs, we should keep in mind the wider context, in particular the adjacent cluster NGC 6611, which has formed thousands of stars within the past few million years (Hillenbrand et al. 1993). The ongoing destruction of the columns and the relatively limited star formation taking place within them may ultimately prove to be a sideshow in the grander scheme of things, albeit a beautiful one.

Acknowledgements. We thank our friends at the VLT who took these excellent data in service mode; Jeff Hester for supplying the original HST images and EGG coordinates; Marc Gagné for 
interesting discussions on M 16; Francesco Palla and Isabelle Baraffe for providing their PMS tracks; and Sandra Jorge and Sofia Fernandes for their independent assessment of the EGGs. This work is supported by the EC RTN "The Formation and Evolution of Young Stellar Clusters" (HPRN-CT-2000-00155) and DLR grants 50-OR-0004/9912.

\section{References}

Andersen, M., Knude, J., Reipurth, B., et al. 2002, A\&A, in press

Baraffe, I., Chabrier, G., Allard, F., \& Hauschildt, P. 1998, A\&A, 337, 403

Bertoldi, F. 1989, ApJ, 346, 735

Cardelli, J. A., Clayton, G. C., \& Mathis, J. S. 1989, ApJ, 345, 245

Chini, R., Krügel, E., \& Wargau, W. F. 1992, A\&A, 265, 45

Currie, D., Kissell, K., Shaya, E., et al. 1996, Messenger, 86, 31

Hester, J. J., Scowen, P. A., Sankrit, R., et al. 1996, AJ, 111, 2349 (H96)

Hillenbrand, L. A., Massey, P., Strom, S. E., \& Merrill, K. M. 1993, AJ, 106, 1906

Hollenbach, D. J., Yorke, H. W., \& Johnstone, D. 2000, in Protostars \& Planets IV, ed. V. Mannings et al. (Tucson: Univ. Arizona Press), 401
Kenyon, S. J., \& Hartmann, L. W. 1995, ApJS, 101, 117

Larosa, T. N. 1983, ApJ, 274, 815

Lefloch, B., \& Lazareff, B. 1994, A\&A, 289, 559

McCaughrean, M. J. 1997, in Herbig-Haro Flows and the Birth of Low Mass Stars, ed. B. Reipurth, \& C. Bertout, IAU Symp., 182, 551

McCaughrean, M. J., \& Andersen, M. 2001, ESO Press Release (http://www.eso.org/outreach/press-rel/pr-2001/ phot-37-01.html)

Palla, F., \& Stahler, S. W. 1999, ApJ, 525, 772

Pilbratt, G. L., Altieri, B., Blommaert, J. A. D. L., et al. 1998, A\&A, 333, L9

Price, S. D., Egan, M. P., Carey, S. J., Mizuno, D. R., \& Kuchar, T. A. 2001, AJ, 121, 2819

Pound, M. W. 1998, ApJ, 493, L113

Schmidt-Kaler, T. 1982, in Landolt-Börnstein, New Series, ed. K. Schaifer, \& H. H. Vogt (Berlin: Springer Verlag), $\mathrm{VI} / 2 \mathrm{~b}, 19$

Sugitani, K., Tamura, M., Nakajima, Y., et al. 2002, ApJ, 565, L25 (S02)

Thompson, R. I., Smith, B. A., \& Hester, J. J. 2002, ApJ, 570, 749 (TSH02)

Walsh, J. R., \& White, N. E. 1982, MNRAS, 199, 9P

White, G. J., Nelson, R. P., Holland, W. S., et al. 1999, A\&A, 342,233 